Trauma Berufskrankh 2008 10 [Suppl 2]:236-240

DOI 10.1007/s10039-007-1320-6

Online publiziert: 26. April 2008

(c) Springer Medizin Verlag 2008

J. Windolf $\cdot$ M. Hakimi · M. Schädel-Höpfner

Klinik für Unfall- und Handchirurgie, Universitätsklinikum, Heinrich-Heine-Universität Düsseldorf

\title{
Ergebnisse der palmaren winkelstabilen Plattenosteosynthese am distalen Radius
}

von einfachen extraartikulären Brüchen bis hin zu komplexen intraartikulären Trümmerfrakturen und erfordert somit entsprechend differenzierte Therapiestrategien. In unserer Klinik hat sich ein abgestuftes Behandlungskonzept auf der Basis der AO-Klassifikation bewährt.

\section{Stufenkonzept}

A2-Frakturen werden ggf. nach Reposition konservativ im Unterarmgipsverband behandelt. Bei kindlichen Frakturen mit sekundärer Dislokationstendenz kann zur Stabilisierung zusätzlich eine KirschnerDraht-Osteosynthese erforderlich werden. Erwachsenen Patienten bieten wir alternativ zur Gipsruhigstellung die Versorgung mit einer palmaren Platte mit dem Ziel einer schnellstmöglichen gipsfreien Nachbehandlung an, um beispielsweise eine frühere Rückkehr an den Arbeitsplatz zu ermöglichen. B1-Frakturen werden nach anatomischer Reposition - ggf. unter arthroskopischer Kontrolle - mittels 3,5er Zugschrauben versorgt. Frakturen vom Typ $\mathrm{A}_{3}, \mathrm{~B}_{2}, \mathrm{~B}_{3}, \mathrm{C}_{1}$ und die meisten $\mathrm{C}_{2}$-Frakturen stabilisieren wir in der Regel mit einer winkelstabilen Plattenosteosynthese von palmar.

\section{Abklärung komplizierter Verletzungen}

Bei Frakturen mit intraartikulärer Trümmerzone, ulnaren Kantenfragmenten oder bei unklarem Frakturverlauf im konventionellen Röntgenbild führen wir präoperativ eine Computertomographie zur genauen Analyse der Verletzung durch. Dislozierte $\mathrm{C}_{3}$-Frakturen werden nach geschlossener Reposition unter Nutzung der Ligamentotaxis zunächst im gelenkübergreifenden Fixateur externe ruhig gestellt und in dieser Stellung einer CT-Diagnostik zugeführt. Eine CT im nicht reponierten Zustand halten wir nicht für aussagefähig.

\section{Operation}

Die definitive Versorgung erfolgt je nach Frakturmorphologie über einen palmaren und/oder einen dorsalen Zugang. Beim dorsalen Vorgehen stabilisieren wir den ulnaren und den radialen Pfeiler mit einer L-förmigen und einer geraden Platte. Ausgedehnte Defekte werden zuvor mit Spongiosa und/oder Knochenersatzmaterial aufgefüllt.

Der Zugang für die palmaren Osteosynthesen erfolgt standardisiert über einen radiopalmaren Zugang. Nach kurzer Längsinzision wird dabei streng radial des M. flexor carpi radialis auf den M. pronator quadratus präpariert und dieser längs inzidiert. Eine routinemäßige Darstellung und Spaltung des Lig. carpi transversum führen wir nicht durch. Nach Komplettierung der Osteosynthese wird bei allen Frakturen mit intraartikulärem Verlauf oder in der präoperativen Bildge- 
bung weitem skapholunären Abstand routinemäßig die Stabilität des Carpus unter Durchleuchtung überprüft.

\section{Postoperative Behandlung}

Postoperativ erfolgt zunächst eine Ruhigstellung mittels dorsaler Unterarmgipsschiene, aus der je nach Frakturtyp und Compliance des Patienten frühzeitig physiotherapeutisch weiterbehandelt wird. Liegt eine karpale Begleitverletzung vor, erfolgt eine Ruhigstellung von bis $\mathrm{zu}$ 6 Wochen.

\section{Patienten und Methode}

In einem Zeitraum von 20 Monaten wurden an unserer Klinik 119 erwachsene Patienten [ 81 Frauen und 38 Männer, Durchschnittsalter 62 (18-94) Jahre] mit einer distalen Radiusfraktur behandelt.

Die Verteilung auf die AO-Klassifikation ist in Abb. 1 dargestellt. Mit 26 C1, $50 \mathrm{C}_{2}$ - und ${ }_{13} \mathrm{C}_{3}$-Frakturen fanden sich in der überwiegenden Mehrzahl der Fälle intraartikuläre Verletzungen. Lediglich 19 Patienten hatten sich eine extraartikuläre $\mathrm{A}_{3}$-Fraktur mit dorsaler Trümmerzone zugezogen. Die Verletzungen von $8 \mathrm{~Pa}$ tienten ließen sich als A2-Fraktur klassifizieren und wurden konservativ behandelt. 6 Patienten konnten sich bei höhergradiger Fraktur (2-mal A3- und 4-mal C1-Frakturen) nicht zu einer operativen Behandlung entschließen, sodass insgesamt 105 Patienten (88\%) zur Operation kamen.

Ein Patient hatte eine B1-Fraktur, die mittels Schraubenosteosynthese stabilisiert wurde. 5 Patienten wurden am Unfalltag geschlossen reponiert und im $\mathrm{Fi}$ xateur externe ruhig gestellt. Bei 2 davon zeigte die anschließende Computertomographie ein ausreichendes Repositionsergebnis, sodass im Fixateur weiterbehandelt werden konnte. 3 Patienten wurden nach Frakturanalyse durch CT im 2. Schritt mittels Plattenosteosynthese über einen kombinierten dorsopalmaren $\mathrm{Zu}$ gang stabilisiert. Die übrigen 99 Patienten wurden primär mit einer winkelstabilen Platte von palmar versorgt. Zum Einsatz kamen hierbei:

- die distale LDR-Radiusplatte 2,4 von Synthes,

Trauma Berufskrankh 2008 · 10[Suppl 2]:236-240 DOI 10.1007/s10039-007-1320-6

(c) Springer Medizin Verlag 2008

\section{J. Windolf $\cdot$ M. Hakimi $\cdot$ M. Schädel-Höpfner Ergebnisse der palmaren winkelstabilen Plattenosteosynthese am distalen Radius}

\section{Zusammenfassung}

Die winkelstabile Plattenosteosynthese von palmar findet zur operativen Behandlung der distalen Radiusfraktur zunehmend Verbreitung, obwohl valide Daten zu ihrer Überlegenheit gegenüber anderen etablierten Verfahren bislang nicht vorliegen. Es werden die Ergebnisse einer prospektiven Beobachtungsstudie vorgestellt und diskutiert. Insgesamt konnten die Behandlungsdaten von 119 Patienten mit distaler Radiusfraktur erhoben werden. Knapp 2/3 davon hatten eine C-Fraktur erlitten. 99 Patienten wurden von palmar mit einer winkelstabilen Plattenosteosynthese stabilisiert. Das funktionelle und radiologische Behandlungsergebnis wurde 12 (1015) Wochen postoperativ im Martini-Score bewertet. $69 \%$ der Patienten wiesen zu diesem Zeitpunkt ein gutes bis sehr gutes Ergeb- nis auf. 9\% der Patienten hatten im Verlauf Komplikationen entwickelt. Die Diskussion der Ergebnisse zielt auf eine sorgfältige und differenzierte Indikationsstellung. Während A3-Frakturen sowie die Mehrzahl der C1- und C2-Brüche in der Regel sicher mit einer winkelstabilen Plattenosteosynthese von palmar versorgt werden können, sind z. B. Frakturen mit dorso-ulnaren Kantenfragmenten, intraartikulären Trümmerzonen bei erhaltener palmarer Kortikalis oder ligamentären Begleitverletzungen durch diese Technik meist nicht ausreichend adressierbar.

\section{Schlüsselwörter}

Distale Radiusfraktur - Winkelstabile palmare Platte - Martini-Score - Komplikationen . Indikationsstellung

\section{Results of internal fixation for unstable distal radius fracture with palmar locking plate}

\section{Abstract}

Fixed-angle palmar plating for fractures of the distal radius is being used more frequently, although valid data are not yet available. In this paper we present the results following treatment of 119 patients with distal radius fractures. In two-thirds of the cases, type $C$ fractures were present. Ninety-nine patients were treated with fixed-angle plates from a palmar approach. Patients were evaluated an average of 12 (range 10-15) weeks postoperatively, both functionally and radiologically, and were assessed using the Martini score. Good or very good results were found in $69 \%$ of cases. Postoperative complications were observed in $9 \%$ of cases. As a re- sult of our study, we advocate a more precise and differentiated indication for palmar locking plate osteosynthesis for distal radial fractures. A3 fractures as well as $\mathrm{C} 1$ and $\mathrm{C} 2$ fractures are good indications for fixed-angle plates. More complicated fractures, such as those with dorsoulnar fragments, intraarticular depression fractures with no cortical lesions, and fractures with concomitant injuries of the carpal ligaments, require other treatment strategies.

\section{Keywords}

Distal radius fracture Palmar locking plate . Martini score $\cdot$ Complications $\cdot$ Indication 


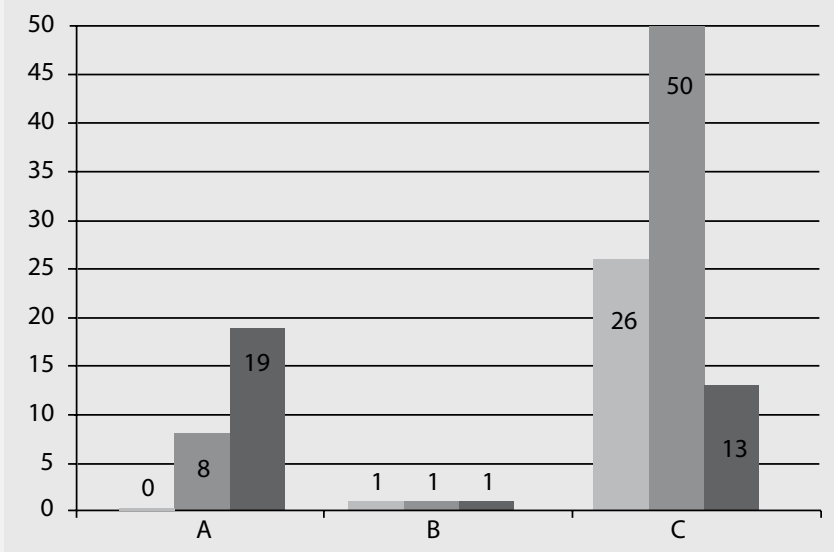

Abb. $1 \Delta$ AO-Klassifikation der 119 Frakturen

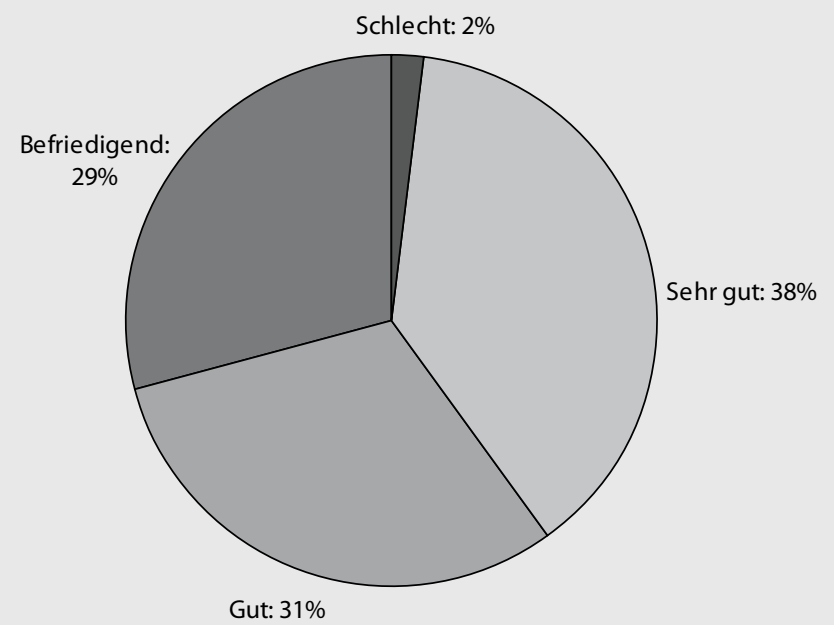

Abb. $2 \Delta$ Ergebnisse im Martini-Score

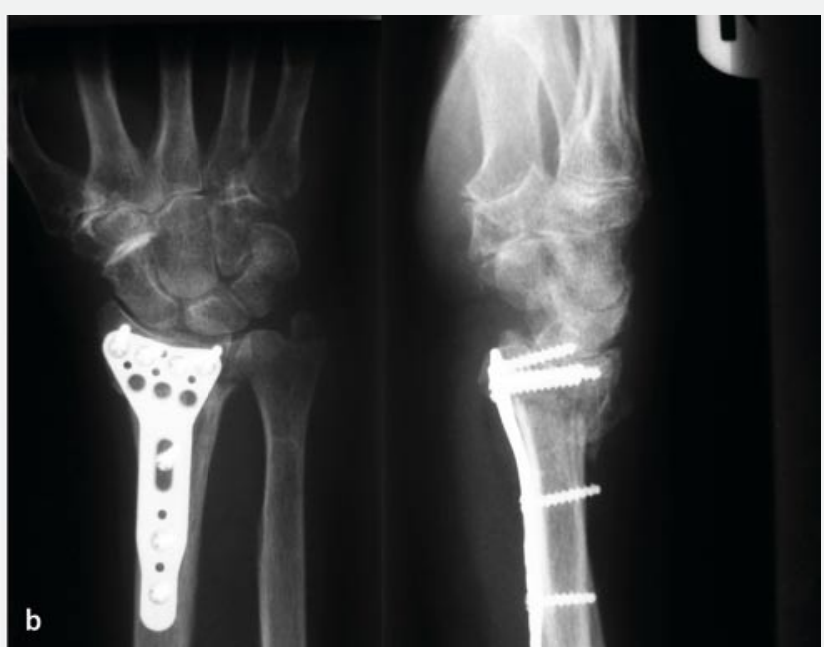

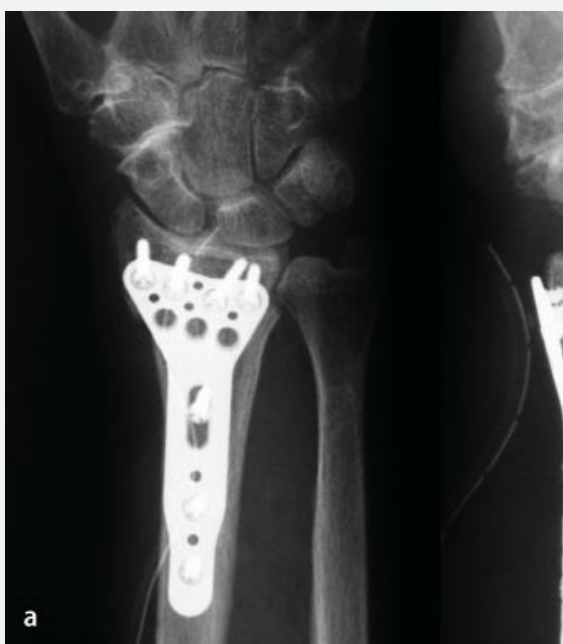

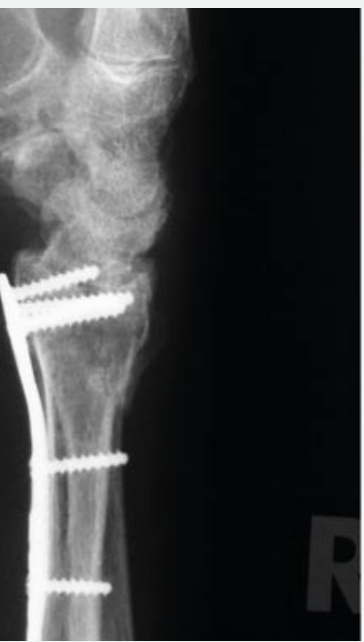

Abb. $3 \Delta$ Sekundärer Korrekturverlust bei einer 81-jährigen Patientin nach palmarer winkelstabiler Plattenosteosynthese einer A3-Fraktur, a postoperatives Ergebnis, b Kontrolle nach 6 Wochen

- die Aptus-Radiusplatte 2,5 von Medartis und

- die Matrix Smartlock 2,7 von Stryker.

Bei 2 Patienten wurde aufgrund eines begleitenden skapholunären Bandschadens eine zusätzliche interkarpale KirschnerDraht-Arthrodese für 8 Wochen erforderlich. Anlässlich der routinemäßigen Abschlussuntersuchung nach 3 Monaten (10-15 Wochen) konnte bei 81 Patienten das radiologische und funktionelle Ergebnis im Martini-Score erhoben werden [5] (• Tab. 1).

\section{Ergebnisse}

Alle Frakturen zeigten bei der Abschlussuntersuchung einen knöchernen Durchbau.

Schmerz. 46 Patienten klagten zum Zeitpunkt der Abschlussuntersuchung noch über Schmerzen bei starker Belastung und 12 über Schmerzen bei Alltagstätigkeiten. Demgegenüber waren 23 Patienten gänzlich schmerzfrei. Unter Ruheschmerzen litt kein Patient.

Kraft. Die grobe Kraft war bereits bei 23 Patienten wieder normal ausgeprägt. 36 Patienten zeigten eine leichte Minderung derselben um bis zu $20 \%$, und $22 \mathrm{~Pa}$ tienten hatten noch eine deutliche Kraft- reduktion von bis zu $50 \%$ im Vergleich zur gesunden Gegenseite.

Beweglichkeit. Die Handgelenkbeweglichkeit war für die Extension und Flexion erst bei lediglich der Hälfte der Patienten wieder nahezu frei. 23 Patienten zeigten eine mäßige und 19 Patienten eine deutliche Einschränkung bis zu $45^{\circ}$. Deutlich besser waren die Ergebnisse für die Unterarmdrehung. Hier wiesen nur 3 Patienten eine erhebliche Einschränkung auf, während 40 Patienten leicht eingeschränkt und 38 Patienten nahezu frei umwenden konnten.

Anatomie. Die Auswertung der Routineröntgenaufnahmen zum Behandlungsabschluss zeigte minimale Verkürzungen 
bzw. Restverkippungen der Gelenkfläche bei 67 bzw. 72 Patienten. Eine relevante Radiusverkürzung bestand bei 14 Patienten.

Gesamtscore. Insgesamt ergab der Martini-Score für $69 \%$ der Patienten ein gutes oder sehr gutes Ergebnis (• Abb. 2), 29\% der Patienten zeigten ein befriedigendes und $2 \%$ ein schlechtes Ergebnis. In der Einzelanalyse der subjektiven Patientenzufriedenheit zeigten sich 3 Patienten mit der Behandlung unzufrieden, während 19 Patienten das Ergebnis befriedigend fanden. 59 Patienten waren mit der Behandlung voll zufrieden.

Insgesamt hatten 9 Patienten einen komplizierten Verlauf:

- 2 Patienten entwickelten postoperativ einen subkutanen Infekt, der allerdings in beiden Fällen konservativ beherrscht werden konnte und nicht $\mathrm{zu}$ einer Revision zwang.

- 4 Patienten klagten postoperativ über Irritationen des $\mathrm{N}$. medianus, 2 davon entwickelten ein manifestes CTS, sodass nachoperiert werden musste.

- Bei 3 Patienten kam es im Verlauf zu einer Sinterung der Fragmente mit sekundärem Korrekturverlust

\section{(- Abb. 3).}

Eine weitere Patientin klagte über Schmerzen im Bereich der nicht gut anmodellierten Platte, was zu einer vorzeitigen Implantatentfernung nach 14 Wochen zwang. Nach diesem 2. Eingriff war die Patientin beschwerdefrei.

\section{Diskussion}

Die winkelstabile Plattenosteosynthese distaler Radiusfrakturen von palmar wird allgemein als einfach anwendbares und sicheres Therapieverfahren beschrieben $[6,7,10]$. Mit zunehmender Verbreitung der Methode mehren sich aber auch die Berichte über die möglichen Komplikationen. Verschiedene Autoren fanden eine Komplikationsrate von bis zu 34\% [1, 2, 3]. Im Vordergrund stehen dabei v. a. Sehnenirritationen und -rupturen. Beugeseitig kann es durch mangelhaft anmodellierte oder zu weit distal platzierte Platten zur Verletzung der M. flexor-pollicis-longusSehne kommen $[2,3]$. Auf der Strecksei-

\begin{tabular}{|c|c|c|c|}
\hline Kriterium & \multicolumn{2}{|l|}{ Ausprägung } & Punkte \\
\hline \multirow[t]{4}{*}{ Schmerz } & \multicolumn{2}{|l|}{ Schmerzfrei } & 10 \\
\hline & \multicolumn{2}{|c|}{ Bei starker Belastung (wie Gartenarbeit, Radwechsel) } & 8 \\
\hline & \multicolumn{2}{|c|}{ Bei Alltagstätigkeit (wie Zähne putzen, Kämmen) } & 4 \\
\hline & \multicolumn{2}{|l|}{ Ruheschmerz } & 0 \\
\hline \multirow[t]{3}{*}{ Subjektives Gesamturteil } & \multicolumn{2}{|l|}{ Voll zufrieden } & 6 \\
\hline & \multicolumn{2}{|l|}{ Befriedigend } & 3 \\
\hline & \multicolumn{2}{|l|}{ Unzufrieden } & 0 \\
\hline \multirow{4}{*}{$\begin{array}{l}\text { Grobe Kraft (im Vergleich zur } \\
\text { gesunden Seite) }\end{array}$} & \multicolumn{2}{|l|}{ Normal } & 4 \\
\hline & \multirow[t]{3}{*}{ Reduktion um } & $10-20 \%$ & 3 \\
\hline & & $21-50 \%$ & 2 \\
\hline & & $>50 \%$ & 0 \\
\hline \multirow[t]{3}{*}{ Arbeit und Sport } & \multicolumn{2}{|l|}{ Keine Einschränkung } & 4 \\
\hline & \multicolumn{2}{|l|}{ Schonung/kein Sport mehr } & 2 \\
\hline & \multicolumn{2}{|l|}{ Arbeitsplatzwechsel } & 0 \\
\hline \multirow{4}{*}{$\begin{array}{l}\text { Bewegung des Handgelenks } \\
(E / F+R / U)\end{array}$} & \multicolumn{2}{|l|}{$>130^{\circ}$} & 4 \\
\hline & \multicolumn{2}{|l|}{$90-129^{\circ}$} & 3 \\
\hline & \multicolumn{2}{|l|}{$45-89^{\circ}$} & 2 \\
\hline & \multicolumn{2}{|l|}{$<45^{\circ}$} & 0 \\
\hline \multirow{4}{*}{$\begin{array}{l}\text { Unterarmdrehung } \\
\text { (Supination und Pronation) }\end{array}$} & \multicolumn{2}{|l|}{$>150^{\circ}$} & 4 \\
\hline & \multicolumn{2}{|l|}{$140-149^{\circ}$} & 3 \\
\hline & \multicolumn{2}{|l|}{$90-139^{\circ}$} & 1 \\
\hline & \multicolumn{2}{|l|}{$<90^{\circ}$} & 0 \\
\hline Röntgenbefund & Radiusverkürzung & $<3 \mathrm{~mm}$ & 2 \\
\hline & & $4-6 \mathrm{~mm}$ & 1 \\
\hline & & $>7 \mathrm{~mm}$ & 0 \\
\hline & Verkippung distale Radiusgelenk- & $<15^{\circ}$ & 2 \\
\hline & fläche (a.-p. und seitlich) & $16-30^{\circ}$ & 1 \\
\hline & & $>30^{\circ}$ & 0 \\
\hline & Distales Radioulnargelenk & Keine Veränderung & 2 \\
\hline & & $\begin{array}{l}\text { Subluxation/Ar- } \\
\text { throse }\end{array}$ & 0 \\
\hline Negativliste & Postoperative Arthrose des Handg & enks & -2 \\
\hline & Pseudarthrose & & -2 \\
\hline & $\begin{array}{l}\text { Reoperation/weitere Behandlung } \\
\text { erforderlich }\end{array}$ & laßnahmen & -2 \\
\hline & Nerven-/Sehnenschädigung & & -2 \\
\hline & Einschränkung der Fingerbewegli & keit & -2 \\
\hline Beurteilung & Sehr gut & & $35-38$ \\
\hline & Gut & & $29-34$ \\
\hline & Befriedigend & & $20-28$ \\
\hline & Schlecht & & $<20$ \\
\hline
\end{tabular}

te sind es insbesondere zu lang gewählte distale Schrauben, die für Rupturen der durch die Fraktur bereits ohnehin gefährdeten M.-extensor-pollicis-longus-Sehne und anderer Strecksehnen verantwortlich gemacht werden $[1,2]$. Überdies wurden postoperative Karpaltunnelsyndrome, Reflexdystrophien, Pseudarthrosen, Korrekturverluste und Implantatlockerungen beschrieben $[2,3,6,7,10]$.
In - Tab. 2 sind die in der jüngsten Literatur publizierten Komplikationsraten in einer Übersicht zusammengefasst. Da randomisierte klinische Studien nicht verfügbar sind, stellen diese Daten neben den berichteten Behandlungsergebnissen die wertvollsten Hinweise auf die Wertigkeit des Verfahrens dar. Ihre Analyse sollte sich auf die Frage nach der etwaigen Vermeidbarkeit der jeweiligen Komplikation konzentrieren. Hierdurch können tech- 
Tab. 2 Komplikationen [\%] der winkelstabilen Plattenosteosynthesen von palmar

\begin{tabular}{|c|c|c|c|c|c|c|c|}
\hline Zitat & $\begin{array}{l}\text { Drobertz u. Kutscha- } \\
\text { Lissberg [3] }\end{array}$ & $\begin{array}{l}\text { Sakhaii } \\
\text { et al. [7] }\end{array}$ & $\begin{array}{l}\text { Thielke } \\
\text { et al. [10] }\end{array}$ & $\begin{array}{l}\text { Al Rashid } \\
\text { et al. [1] }\end{array}$ & $\begin{array}{l}\text { Rozental } \\
\text { u. Blazar [6] }\end{array}$ & $\begin{array}{l}\text { Arora } \\
\text { et al. [2] }\end{array}$ & Eigene \\
\hline Jahr & 2003 & 2003 & 2003 & 2006 & 2006 & 2007 & 2007 \\
\hline Anzahl Patienten & 50 & 100 & 49 & 35 & 41 & 114 & 99 \\
\hline Wundheilungsstörung [\%] & 8 & 1 & 1 & - & - & 0 & 2 \\
\hline Ruptur der M. flexor-pollicis-longus-Sehne [\%] & 12 & - & 0 & - & - & 3 & 0 \\
\hline Ruptur der M.-extensor-pollicis-longus-Sehne [\%] & 2 & 2 & - & 9 & - & 2 & 0 \\
\hline Karpaltunnelsyndrom [\%] & 2 & 2 & 0 & - & - & 3 & 4 \\
\hline Implantatversagen [\%] & 2 & 16 & - & - & - & 4 & 2 \\
\hline Reflexdystrophie [\%] & 6 & 2 & 4 & - & - & 4 & 0 \\
\hline Frakturheilungsstörung [\%] & - & 3 & - & - & 7 & 3 & 0 \\
\hline Sonstige [\%] & 2 & 1 & 7 & - & 7 & 8 & 1 \\
\hline Gesamt [\%] & 34 & 27 & 12 & 9 & 14 & 27 & 9 \\
\hline
\end{tabular}

nische Fehler limitiert und ungeeignete Indikationen identifiziert werden. In einigen Fallserien trat ein Implantatversagen mit bis zu $16 \%$ relativ häufig auf [7]. Unseres Erachtens ist dies ein Hinweis darauf, dass die Indikation zur winkelstabilen Versorgung von palmar zu weit gestellt wurde. So eignen sich beispielsweise Frakturen mit intraartikulärer Trümmerzone und intakter palmarer Kortikalis nicht zu einer Reposition von palmar. Ebenso sollten mühsam rekonstruierte Gelenkflächen bei osteoporotischem Knochen auch bei winkelstabiler Versorgung nicht zu früh funktionell weiterbehandelt werden.

Für die häufigsten Frakturtypen am distalen Radius unterstreichen die hier vorgelegten Daten aber eindrucksvoll, dass durch die winkelstabile Plattenosteosynthese von palmar gute bis sehr gute Ergebnisse erzielbar sind. Die in der Literatur beschriebene hohe Komplikationsrate lässt sich durch sorgfältige Analyse der berichteten Probleme und eine differenzierte Indikationsstellung deutlich senken. Dabei sollte berücksichtigt werden, dass nicht alle Frakturtypen für eine palmare Versorgung geeignet sind. Insbesondere $\mathrm{C}_{3}$-Verletzungen erfordern häufig ein kombiniertes dorso-palmares Vorgehen und ggf. auch eine Defektauffüllung. Die palmare Versorgung ist nur für Frakturen mit ausreichend großen distalen Fragmenten geeignet.

Die klinische Wertigkeit des Verfahrens wird sich langfristig in weiteren klinischen Studien und durch eine offene und kritische Diskussion der Ergebnisse zeigen.

\section{Fazit für die Praxis}

Die palmare winkelstabile Plattenosteosynthese führt bei sachgerechter Indikationsstellung zu guten funktionellen und radiologischen Ergebnissen. Die Komplikationsrate kann durch eine sorgfältige Operationstechnik gesenkt werden. Verletzungen der Sehnen lassen sich durch eine korrekte Schraubenlänge und exakte Platzierung der Platte vermeiden. Frakturen mit intraartikulären Trümmerzonen oder sehr kleinen distalen Fragmenten sind für eine Versorgung von palmar nur sehr eingeschränkt geeignet.

\section{Korrespondenzadresse}

Prof. Dr. J. Windolf

Klinik für Unfall- und Handchirurgie,

Universitätsklinikum,

Heinrich-Heine-Universität Düsseldorf,

Moorenstraße 5, 40225 Düsseldorf

windolf@uni-duesseldorf.de

Interessenkonflikt. Der korrespondierende Autor gibt an, dass kein Interessenkonflikt besteht.

\section{Literatur}

1. Al-Rashid M, Theivendran K, Craigen MAC (2006) Delayed ruptures of the extensor tendon secondary to the use of volar locking compression plates for distal radial fractures. J Bone Joint Surg Br 88: 1610-1612

2. Arora R, Lutz M, Hennerbichler A et al. (2007) Complications following internal fixation of unstable distal radius fracture with a palmar locking-plate. J Orthop Trauma 21: 316-322

3. Drobetz H, Kutscha-Lissberg E (2003) Osteosynthesis of distal radial fractures with a volar locking screw plate system. Int Orthop 27: 1-6
4. Koshimune M, Kamano M, Takamatsu K et al. (2005) A randomized comparison of locking and non-locking palmar plating for unstable Colles' fractures in the elderly. J Hand Surg $[\mathrm{Br}] 30 \mathrm{~B}-5$ : 499-503

5. Martini AK (1999) Bewertungsschemata zur Beurteilung des Handgelenkes. Handchir Mikrochir Plast Chir 31: 153-154

6. Rozental TD, Blazar PE (2006) Functional outcome and complications after volar plating for dorsally displaced, unstable fractures of the distal radius. J Hand Surg [Am] 31:359-365

7. Sakhaii M, Groenewold U, Klonz A et al. (2003) Ergebnisse nach palmarer Plattenosteosynthese mit der winkelstabilen T-Platte bei 100 distalen Radiusfrakturen. Eine prospektive Studie. Unfallchirurg 106: $272-280$

8. Schädel-Höpfner M, Diener M, Windolf J (2007) Gesicherte Literaturergebnisse zur Therapie distaler Radiusfrakturen. Trauma Berufskrankh $\otimes$

9. Schupp A, Tuttlies C, Möhlig T et al. (2003) Der distale Speichenbruch. Winkelstabile Osteosynthese mit $2.4 \mathrm{~mm}$ Formplatten. Ist der Aufwand gerechtfertigt? Chirurg 74: 1009-1017

10. Thielke KH, Wagner T, Bartsch S et al. (2003) Winkelstabile, volare Plattenosteosynthese komplexer artikulärer Frakturen am distalen Radius. Lösung einer Prolemfraktur? Chirurg 74: 1057-1063 